\title{
Funções densidade de probabilidade para a estimativa de precipitação mensal
}

\section{Probability density functions for the estimation of monthly rainfall}

\author{
Jailson de Araujo Rodrigues ${ }^{1}$; Jaime dos Santos Filho ${ }^{2}$; Lucas Monteiro Chaves ${ }^{3}$
}

\section{Resumo}

O objetivo deste trabalho foi avaliar o potencial das distribuições gama, log-normal e Weibull na modelagem de precipitação mensal na cidade de Bento Gonçalves, Rio Grande do Sul. Foi utilizada uma série histórica de precipitações pluviométricas mensais no período de janeiro de 2001 a dezembro de 2011. A aderência das distribuições às condições pluviométricas foi verificada via teste de KolmogorovSminorv. As três distribuições apresentaram aderência. Entretanto, os resultados obtidos indicaram que o modelo gama ajustou-se mais adequadamente às condições pluviométricas da cidade de Bento Gonçalves.

Palavras-chave: Comparação de Modelos. Distribuição Gama. Distribuição Log-normal. Distribuição Weibull.

\begin{abstract}
The objective of this work was to evaluate the potential of gamma, log-normal and Weibull distributions modeling of monthly precipitation in the city of Bento Gonçalves, Rio Grande do Sul. We used a series of monthly rainfall from January 2001 to December 2011. Adherence to the distributions of rainfall conditions was verified using the Kolmogorov-Sminorv test. The three distributions were adherent. However, the results indicated that the gamma model fit better rainfall conditions in the city of Bento Gonçalves.

Keywords: Comparison of Models. Gamma Distribution. Lognormal Distribution. Weibull Distribution.
\end{abstract}

\section{Introdução}

Medir dados de precipitação pluviométrica é muito importante em diversos contextos, tais como produtividade de culturas agrícolas, manejo dos recursos hídricos, avaliação ambiental, erosão hídrica, etc. A obtenção da correta distribuição temporal para precipitação é relevante no planejamento agrícola, no que diz respeito à instalação de culturas. Além da influência na agricultura, períodos de estiagens muito longos afetam o nível de água dos mananciais e dos reservatórios das usinas hidrelétricas, trazendo problemas para o abastecimento urbano e na geração de energia elétrica. Segundo Vieira e Carvalho (2001), a quantificação das chuvas é importante no planejamento agrícola e ambiental para o correto dimensionamento das obras, tanto na construção civil quanto na conservação do solo.

${ }^{1}$ Docente do Instituto Federal da Bahia; jailsondearaujo@yahoo.com.br.

${ }^{2}$ Discente do Doutorado em Estatística e Experimentação Agropecuária, UFLA: jsf_ba@yahoo.com.br.

${ }^{3}$ Docente do Departamento de Ciências Exatas, UFLA: lucasi@dex.edu.br. 
O papel das precipitações pluviométricas é destacado por Botelho e Morais (1999) como principal fonte de água para a agricultura, que por vezes tem comprometido o desenvolvimento da produção agrícola em função de sua variabilidade não uniforme, ora com grandes períodos de estiagem, ora com consideráveis aguaceiros de curta duração, que superam a capacidade de retenção de água pelo solo, provocando enchentes e inundações. A impossibilidade de saber qual a evolução exata dos valores de precipitação ao longo do tempo e do espaço em função de sua natureza aleatória é alertada por Sampaio et al. (2007). A partir dessas dificuldades, diferentes modelos probabilísticos são utilizados para descrever o comportamento tanto da precipitação esperada como do período de precipitação.

Longo et al. (2006) avaliaram o uso das distribuições gama e log-normal na estimativa de precipitações pluviais quinzenais no Estado do Paraná, a aderência dos modelos foi verificada via teste qui-quadrado e teste de Kolmogorov-Smirnov. Os resultados mostraram que a distribuição gama obteve melhor ajuste das condições pluviométricas do Estado.

Uma generalização do modelo gama foi introduzida por Nadarajah e Gupta (2007) para estudar a intensidades de secas ocorridas no Estado de Nebraska. Os resultados indicaram que a nova distribuição fornecia um excelente ajuste para os dados de seca, melhor que o ajuste obtido com o modelo gama usual.

Nadarajah e Yang (2006) estudaram a viabilidade da distribuição do produto $X Y$ de variáveis aleatórias quando $\mathrm{X}$ é exponencialmente distribuída e Y é proveniente da família gama, Weibull, Pareto e log-normal na descrição da magnitude de secas ocorridas em Nebraska-EUA.

Sampaio et al. (2007) através dos modelos gama e log-normal estudaram a distribuição da precipitação mensal para o Estado do Paraná. Os resultados obtidos indicaram que a distribuição gama ajustou- se mais adequadamente à pluviometria do Estado.

Uma análise comparativa entre os modelos gama, Weibull, normal, exponencial e log-normal no ajuste a dados reais de precipitações em Santa Maria no Rio Grande do Sul foi feita por Silva et al. (2007), as distribuições gama e Weibull foram as que apresentaram melhores resultados na descrição da variação da probabilidade de ocorrência de precipitação diária.

A distribuição da combinação linear de variáveis gama e Rayleigh foi utilizada por Shakil e Kribia (2009) para modelar dados de precipitações. A boa qualidade de ajuste do modelo foi verificada através do teste qui-quadrado de aderência.

Rodrigues, Silva e Chaves (2011) utilizaram a distribuição gama para modelar o período de dias com ocorrência de precipitações pluviométricas. A base de dados analisada correspondia às medições diárias do índice pluviométrico na cidade de Passo Fundo no Estado do Rio Grande do Sul. Critérios gráficos indicaram o bom ajuste do modelo em relação aos dados de precipitação analisados.

O objetivo deste trabalho foi avaliar as distribuições gama, log-normal e Weibull na descrição da precipitação pluviométrica mensal na cidade de Bento Gonçalves no Rio Grande do Sul, verificando-se as respectivas aderências por meio do teste de Kolmogorov-Smirnov à nível de 0,05 de probabilidade. A escolha do modelo que apresenta melhor ajuste foi feita via Critério de Informação de Akaike, gráfico P-P plot e p-valores do teste de Kolmogorov-Smirnov.

\section{Materiais e Métodos}

Os dados explorados foram coletados na estação meteorológica da Embrapa Uva e Vinho com sede local em Bento Gonçalves no nordeste do Estado do Rio Grande do Sul. A estação climatológica está situada à latitude de $29^{\circ} 09^{\prime} 44^{\prime \prime} \mathrm{S}$, longitude de $51^{\circ} 31^{\prime} 50^{\prime \prime} \mathrm{W}$ e altitude de $640 \mathrm{~m}$. As observações 
correspondem às medições mensais do índice pluviométrico no período de janeiro de 2001 a dezembro de 2011.

Neste estudo realizou-se o ajuste das distribuições gama, log-normal e Weibull (JOHNSON; KOTZ; BALAKRISHNAN, 1995).

Modelo gama: Uma variável aleatória $X$ tem distribuição gama com parâmetros $\alpha>0$ e $\beta>0$ quando sua função densidade de probabilidade (fdp) é:

$$
f(x)=\frac{\beta^{\alpha} x^{\alpha-1}}{\Gamma(\alpha)} \exp (-\beta x)
$$

em que $x>0$ e $\Gamma(\bullet)$ representa função gama,

$$
\Gamma(\alpha)=\int_{0}^{\infty} t^{\alpha-1} \exp (-t) d t
$$

Se $X_{1}, \ldots, X_{n}$ é uma amostra aleatória de (1), as equações de máxima verossimilhança de $\alpha$ e $\beta$ são:

$$
\begin{gathered}
n \log \left(\frac{n \hat{\alpha}}{\sum_{i=0}^{n} x_{i}}\right)-n \psi(\hat{\alpha})+\sum_{i=0}^{n} \log x_{i}=0 \\
\hat{\beta}=\frac{n \hat{\alpha}}{\sum_{i=0}^{n} x_{i}}
\end{gathered}
$$

em que $\psi(\cdot)_{\text {é a função digama }}$ $\psi(\alpha)=d \log (\alpha) / d \alpha$.

Modelo log-normal: Uma variável aleatória $X$ tem distribuição log-normal com parâmetros $-\infty<\mu<+\infty$ e $\sigma>0$ quando sua fdp é:

$$
f(x)=\frac{1}{x \sigma \sqrt{2 \pi}} \exp \left[\frac{-(\log x-\mu)^{2}}{2 \sigma^{2}}\right]
$$

em que $x>0$.
Se $X_{1}, \ldots, X_{n}$ é uma amostra aleatória de (5), as estimativas de máxima verossimilhança de $\mu \mathrm{e}$ $\sigma^{2}$ são:

$$
\hat{\mu}=\frac{1}{n} \sum_{i=0}^{n} \log x_{i}
$$

$\hat{\sigma}^{2}=\frac{1}{n} \sum_{i=0}^{n}\left(\log x_{i}-\frac{1}{n} \sum_{i=0}^{n} \log x_{i}\right)^{2}$

Modelo Weibull: Uma variável aleatória $X$ tem distribuição Weibull com parâmetros $\alpha>0$ e $\beta>0$ quando sua fdp é:

$f(x)=\frac{\alpha}{\beta}\left(\frac{x}{\beta}\right)^{\alpha-1} \exp \left[-\left(\frac{x}{\beta}\right)^{\alpha}\right]$

em que $x>0$.

Se $X_{1}, \ldots, X$ é uma amostra aleatória de (8), as estimativas de máxima verossimilhança de $\alpha$ e $\beta$ são:

$$
\begin{gathered}
\hat{\beta}=\left(\frac{1}{n} \sum_{i=0}^{n} x_{i}^{\hat{\alpha}}\right)^{1 / \hat{\alpha}} \\
\hat{\alpha}=\left[\left(\sum_{i=0}^{n} x_{i}^{\hat{\alpha}} \log x_{i}\right)\left(\sum_{i=0}^{n} x_{i}^{\hat{\alpha}}\right)^{-1}-\frac{1}{n} \sum_{i=0}^{n} \log x_{i}\right]^{-1}
\end{gathered}
$$

Para verificar a aderência das distribuições descritas, foi utilizado o teste estatístico de Kolmogorov-Smirnov, que além de não depender do número de classes do agrupamento dos dados, oferece mais vantagens computacionais. Esse teste fundamenta-se na discrepância entre as distribuições, $D_{n}=\sqrt{n} \sup _{x}\left|F_{n}(x)-F_{0}(x)\right|$ em que $F_{n}(x)$ denota a distribuição teórica e $F_{0}(x)$ a distribuição ajustada. Essa estatística é usada para testar a hipótese nula $H_{o}: F=F_{0}$ versus hipótese alternativa $H_{l}: F \neq F_{0}$ (KVAM; VIDAKOVIC, 2007). 
Ao trabalharmos com modelos é necessário que internalizemos sua condição de aproximação da realidade, ou seja, não existem modelos probabilísticos exatos, sempre haverá perda de informações, fato inerente à presença da componente de aleatoriedade. Dessa forma, é necessário selecionar o "melhor" modelo dentre aqueles utilizados no ajuste de dados. Burnham e Anderson (2004) enfatizam que tal escolha deve ser embasada em princípios científicos. Dentre as metodologias empregadas para esse fim, uma das mais utilizadas é o critério de informação de Akaike (AIC) estabelecido por Akaike (1983), baseado na teoria de decisão, o AIC é definido como a quantidade:

$$
A I C=-2 L+2 p
$$

em que $L$ representa o magnitude máxima da função suporte e $p$ denota o número de parâmetros. De acordo com esse critério, o melhor modelo é aquele que apresenta o menor valor de AIC.

A qualidade de ajuste das distribuições também foi verificada utilizando os gráficos de probabilidade, os P-P plots. Esses gráficos consistem em representar no plano cartesiano a probabilidade observada versus a probabilidade estimada (esperada) de determinada distribuição. O procedimento de construção desses gráficos é feito utilizando os seguintes passos:

1) Dada uma amostra aleatória $X_{1}, \ldots, X_{n}$, rearrumar a amostra em ordem crescente, $X_{(1)}, \ldots, X_{(n)}$;

2) Determinar as probabilidades observadas $\widetilde{P}_{i}=F\left(x_{(i)}\right)$ para $i=1, \ldots, n$ em que $F(\cdot) \quad$ é a distribuição proposta;

3) Determinar as probabilidades acumuladas $P_{i}=(i-c) /(n-2 c+1) \quad$ correspondentes ao valor da função de distribuição teórica para $x_{(i)}$ em que $i=1, \ldots, n$, representa uma estimativa da função de distribuição no ponto $x_{(i)}$. O valor da constante $c$ pode variar para as diferentes distribuições;
4) Plotar o gráfico de dispersão para os pares de pontos $\left(\widetilde{P}_{i}, P_{i}\right)$ em que $i=1, \ldots, n$.

Se a pressuposição sobre a distribuição está correta os pares de pontos estarão dispostos sobre uma linha reta, ou próximos dela.

\section{Resultados e Discussão}

A precipitação média mensal observada para a cidade de Bento Gonçalves foi de 151,814mm. Observou-se uma variabilidade muito grande nos dados de precipitações mensais, com medição mínima de $17,100 \mathrm{~mm}$, máxima de $417,800 \mathrm{~mm}$ e erro padrão de $83,091 \mathrm{~mm}$.

Tabela 1 - Estimativas dos parâmetros das distribuições gama, log-normal e Weibull.

\begin{tabular}{lcccc}
\hline Modelo & $\hat{\alpha}$ & $\hat{\beta}$ & $\hat{\mu}$ & $\hat{\sigma}^{2}$ \\
\hline Gama & 3,264 & 0,022 & $\ldots .$. & $\ldots .$. \\
Log-normal & $\ldots$. & $\ldots$ & 4,862 & 0,596 \\
Weibull & 1,945 & 171,796 & $\ldots$ & \\
\hline
\end{tabular}

Fonte: Elaborada pelo autor.

Na Tabela 1 podem ser observadas as estimativas de máxima verossimilhança encontradas nos ajustes das distribuições gama, Weibull e log-normal, calculadas utilizando o programa R (versão 2011). As fdp ajustadas podem ser visualizadas na Figura 1 .

Figura 1 - Histograma de freqüência dos dados observados de precipitação mensal sobreposto pelas fdp ajustadas gama, log-normal e Weibull.

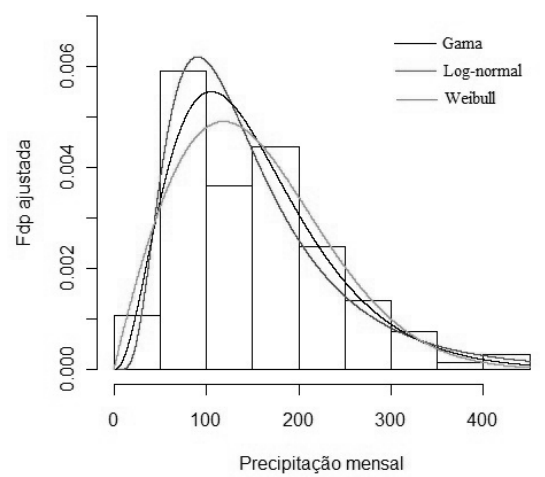

Fonte: Elaborada pelo autor. 
Ao observar a Figura 1, pode-se notar que as curvas de ajustes das três distribuições acompanharam as condições de moda e assimetria, não sendo possível determinar, visualmente, a que melhor se ajustou aos dados observados.

Tabela 2 - Valores de p do teste de KolmogorovSmirnov e AIC.

\begin{tabular}{lcc}
\hline Modelo & p-valor & AIC \\
\hline Gama & 0,642 & 1519,356 \\
Log-normal & 0,264 & 1525,613 \\
Weibull & 0,575 & 1522,017 \\
\hline
\end{tabular}

Fonte: Elaborada pelo autor.

A aderência das distribuições foi comprovada segundo o teste de Kolmogorov-Smirnov, todos os p-valores foram superiores a 0,05 indicando que as distribuições descrevem satisfatoriamente os dados observados de precipitação, ver Tabela 2. Em particular, a distribuição gama foi a que obteve maior aderência seguida da distribuição Weibull e a que obteve menor aderência foi à distribuição lognormal.

Os resultados obtidos no teste de KolmogorovSmirnov foram confirmados pelos respectivos valores obtidos para os AIC's que podem ser visualizados na Tabela 2. A distribuição gama foi a que teve o melhor desempenho, com o menor AIC em relação aos demais. O maior AIC foi da distribuição log-normal confirmando o status de modelo menos adequado.

Os P-P plots para as três fdp ajustadas são exibidos nas Figuras 2 a 4 .

Figura 2 - P-P plot da fdp gama ajustada.

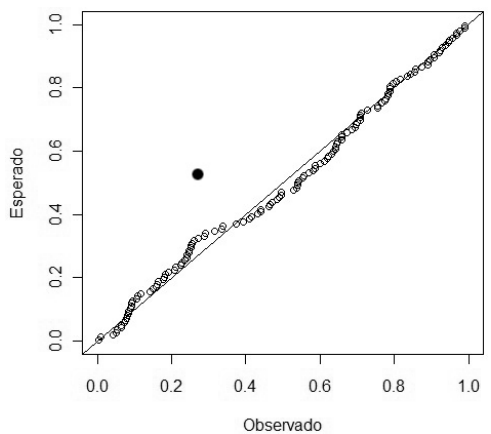

Fonte: Elaborada pelo autor.
Figura 3 - P-P plot da fdp log-normal ajustada.

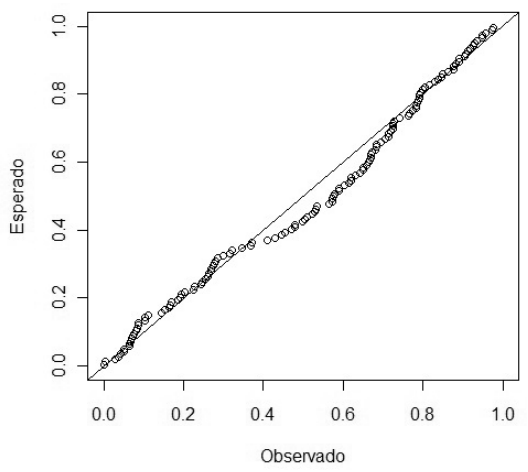

Fonte: Elaborada pelo autor.

Figura 4 - P-P plot da fdp Weibull ajustada.

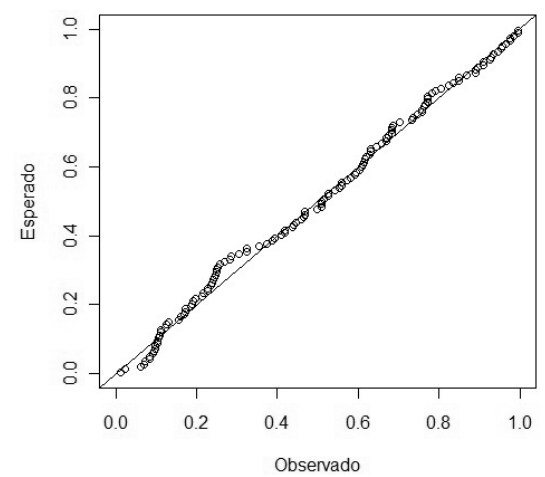

Fonte: Elaborada pelo autor.

Ao observar as Figuras de 2 a 4, pode-se notar que a distribuição log-normal foi a que pior se ajustou em relação aos dados observados e os gráficos referentes às distribuições gama e Weibull indicam um bom ajuste. Entretanto, os gráficos são inconclusivos em relação a que apresenta melhor ajuste. Dessa forma, o critério gráfico de P-P plot acompanhou de certa forma os resultados obtidos anteriormente via teste de Kolmogorov-Smirnov e o Critério de Informação de Akaike.

\section{Conclusões}

O teste de Kolmogorov-Smirnov indicou a aderência das distribuições gama, log-normal e Weibull no nível de 0,05 de significância. No entanto, a distribuição gama foi a que obteve maior aderência e em seguida a distribuição Weibull. A 
distribuição log-normal foi a que apresentou menor aderência.

O Critério de Informação de Akaike confirmou os resultados obtidos pelo teste de KolmogorovSmirnov, indicando o modelo gama como o mais adequado na descrição dos dados pluviométricos de Bento Gonçalves. O modelo de pior desempenho segundo esse critério foi o log-normal.

Os P-P plots também indicaram que as três distribuições descrevem adequadamente a série de precipitações. No entanto, foi inconclusivo com respeito ao melhor ajuste, indicando apenas que a distribuição log-normal é a de pior ajuste em relação aos dados.

\section{Referências}

AKAIKE, H. Information measures and model selection. International Statistical Institute, Netherlands, v. 44, p. 277-291, 1983.

BOTELHO, V. A. V. A.; MORAIS, A. R. Estimativas dos parâmetros da distribuição gama de dados pluviométricos de Município de Lavras, Estado de Minas Gerais. Ciência e Agrotecnologia, Lavras, v. 23, n. 3, p. 697-706, 1999.

BURNHAM, K. P.; ANDERSON, D. R. Multimodel inference: understanding aic and bic in model selection. Sociological Methods and Research, Beverly Hills, v. 33, n. 2, p. 261-304, 2004.

LONGO, J. L.; SAMPAIO, C. S.; QUEIROZ, M. M. F.; SUSZEK, M. Uso das distribuições gama e log-normal na estimativa de precipitação provável quinzenal. Revista Varia Scientia, Cascavel, v. 6, n. 3, p. 107-118, 2006.

NADARAJAH, S.; GUPTA, A. K. A generalized gamma distribution with application to drought data. Mathematics and Computers in Simulation, Amsterdam, v. 74, p. 1-7, 2007.

NADARAJAH, S.; YANG, D. W. Drought modeling and products of random variables with exponential kernel. Stochastic Environmental Research and Risk Assessment, New York, v. 21, n. 2, p. 123-129, 2006.

JOHNSON, N. L.; KOTZ, S.; BALAKRISHNAN, N. Continuous univariate distributions. New York: John Wiley \& Sons, 1995.

KVAM, P. H.; VIDAKOVIC, B. Nonparametric statistics with applications to science and engineering. New York: Wiley, 2007.
R DEVELOPMENT CORE TEAM. R: A language and environment for statistical computing. R Foundation for

Statistical Computing, Vienna, Austria. ISBN 3-90005107-0, URL www.R-project.org. 2011.

RODRIGUES, J. A.; SILVA, A. P. C. M.; CHAVES, L. M. Distribuição gama bivariada de Smith, Adelfang e Tubbs: soma, produto e proporção das variáveis componentes. Revista Ciências Exatas e Naturais, Guarapuava, v. 13, n. 2, p. 185-200, 2011.

SAMPAIO, S. C.; LONGO, A. J.; QUEIROZ, M. M. F.; GOMES; B. M.; BOAS, M. A.; SUSZEK; M. Estimativa e distribuição da precipitação mensal provável no Estado do Paraná. Irriga, Botucatu, v. 12, p. 38-53, 2007.

SHAKIL, M.; KRIBIA, B. M. G. Exact distributions of the linear combination of gamma and Rayleigh random variables. Austrian Journal of Statistics, Sidney, v. 38, p. 33-44, 2009.

SILVA, J. C.; HELDWEIN, A.; MARTINS, F. B.; TRENTIN, G.; GRIMM, E. L. Análise de distribuição de chuva para Santa Maria, RS. Revista Brasileira de Engenharia Agrícola e Ambiental, Campina Grande, v. 11, n. 1, p. 67-72, 2007.

VIEIRA, S. R.; CARVALHO, J. R. P. Estudo da periodicidade temporal de chuvas em uma bacia hidrográfica dos Rios Turvo/Grande - uma proposta. Campinas: Embrapa Informática Agropecuária, 2001. v. 10.

Recebido em 31 Janeiro 2012-Received on January 9, 2012. Aceito em 13 Dezembro, 2012 - Accepted on December 13, 2012. 